\title{
Les métaphores dans la publicité scientifique et technique : de la médiation à l'interaction
}

\section{Marie-Hélène Fries-Verdeil}

\section{(2) OpenEdition}

1 Journals

Édition électronique

URL : http://journals.openedition.org/asp/1973

DOI : 10.4000/asp.1973

ISBN : 978-2-8218-0384-8

ISSN : 2108-6354

\section{Éditeur}

Groupe d'étude et de recherche en anglais de spécialité

Édition imprimée

Date de publication : 1 octobre 2001

Pagination : 227-237

ISSN : 1246-8185

\section{Référence électronique}

Marie-Hélène Fries-Verdeil, « Les métaphores dans la publicité scientifique et technique : de la médiation à l'interaction », ASp [En ligne], 31-33 | 2001, mis en ligne le 22 novembre 2010, consulté le 09 septembre 2020. URL : http://journals.openedition.org/asp/1973 ; DOI : https://doi.org/10.4000/ asp. 1973

Ce document a été généré automatiquement le 9 septembre 2020.

Tous droits réservés 


\title{
Les métaphores dans la publicité scientifique et technique : de la médiation à l'interaction
}

\author{
Marie-Hélène Fries-Verdeil
}

\section{Introduction}

1 Le présent article aborde les métaphores d'un point de vue un peu inhabituel. Tout d'abord, les figures de style y sont étudiées dans un cadre non linguistique, celui des images publicitaires à caractère scientifique ou technique, en partant du principe que les métaphores sont fondamentalement conceptuelles (Lakoff \& Johnson 1980, 1999) ${ }^{1}$. Si on commence par définir étymologiquement les métaphores comme un "transport ", un transfert de sens, on implique nécessairement une trajectoire, une médiation, entre un point de départ et un point d'arrivée.

2 On peut donc poser que le concept de médiation peut aider à cerner le fonctionnement des métaphores. Dans le contexte des publicités à caractère scientifique ou technique, ce fonctionnement est doublement inséparable de la persuasion. D'une part, en effet, historiquement Aristote a choisi de placer son analyse des métaphores partiellement sous l'égide de la rhétorique, considérée par les Grecs comme l'art de la persuasion. D'autre part, pour comprendre les métaphores il faut prendre en compte leur contexte publicitaire : le choix d'un public-cible, les contraintes économiques et financières, et, bien sûr, les stratégies de persuasion de l'annonceur.

Dans cette double perspective, à la fois historique et pragmatique ${ }^{2}$, on peut formuler l'hypothèse selon laquelle chaque théorie de la métaphore, même celles dont les limites sont démontrées depuis longtemps peut aider à comprendre la nature de la médiation à l'œuvre dans telle ou telle métaphore publicitaire, dans la mesure où elle rend explicite la stratégie de persuasion affichée. Dans une perspective historique, cette hypothèse va être testée sur deux approches traditionnelles, la théorie comparative d'Aristote et la 
théorie substitutive de l'époque moderne, et une approche récente, la théorie de l'interaction de Max Black.

\section{La théorie de la comparaison (Aristote) : fonction analogique}

Aristote, dans sa Poétique (ch. 21 et 22) et dans sa Rhétorique (livre 3), a été à l'origine de la théorie comparative de la métaphore, qui privilégie la similitude comme critère de reconnaissance et d'explication. «Car bien métaphoriser, c'est bien apercevoir les ressemblances » (Poétique 1459 a).

5 Aristote distingue plusieurs sortes de métaphores, dont la métaphore proportionnelle ou rapport d'analogie qui nous intéresse directement, parce qu'elle exhibe clairement une médiation, sous forme de ressemblance. Et cette ressemblance fait l'objet d'une construction méthodique qui peut se schématiser sous la forme d'une égalité entre deux ratios de deux termes:

Il faut toujours, dans la métaphore par analogie, observer le rapport réciproque de deux termes appartenant au même genre; par exemple, si la coupe est le bouclier de Dionysos, la concordance exige que le bouclier soit appelé la coupe d'Arès. (Rhétorique, III, 4, 15-18)

6 Dans l'exemple mythologique choisi par Aristote, la coupe symbolise Dionysos, dieu grec de la vigne et du vin, tout comme le bouclier Arès, dieu de la guerre. Sur la base de l'égalité entre les deux ratios, la métaphore consiste alors à substituer le quatrième terme au second ( le bouclier de Dionysos ») ou vice versa (« la coupe d'Arès »).

7 L'analyse aristotélicienne, qui a eu beaucoup de succès jusqu'au dix-neuvième siècle, est-elle toujours pertinente? Par exemple, prenons une publicité pour Sony publiée dans le magazine américain Discover en décembre 1999 (annexe 1), qui montre en gros plan une tête de jeune homme aux cheveux coupés ras, vue de derrière. Sur le crâne, entre les deux oreilles, a été greffé un lecteur informatique dont on voit sortir une carte «memory stick». Le caractère hybride de ce personnage, mi-homme, mi-machine, est souligné par un argumentaire publicitaire énigmatiquement court : «think it». Le neutre « it » semble a priori renvoyer au lecteur informatique, alors que «think» nous rappelle comment, depuis Descartes et son cogito, la pensée est le propre de l'être humain en général, et, plus particulièrement, du concepteur de cette innovation, ou du lecteur de cette publicité. Mais la présence de la greffe peut aussi autoriser la lecture inverse : et si «think» renvoyait à l'intelligence artificielle symbolisée par la carte, la tête (et, par synecdoque ${ }^{3}$, l'être humain tout entier) étant alors transformée en ordinateur vivant? Seul le contexte permet de dissiper l'ambiguïté. En fait, cette publicité fait partie d'un quadriptyque: «See it, hear it, think it, stick it». Les trois premiers volets présentent une version anthropomorphe d'un appareil Sony (la première image nous montre une tête de jeune fille dont les yeux ont été remplacés par des objectifs de caméscope ou d'appareil photo, la seconde une jeune femme de profil dont l'oreille est décorée avec les touches de commande d'un appareil hi-fi), et le dernier volet révèle comment tous ces appareils peuvent se connecter au sein du système "memory stick». La greffe informatique peut alors s'interpréter sous forme d'un ratio aristotélicien : un ordinateur est au système "memory stick» Sony ce que le cerveau est au corps humain: tous deux permettent de "penser». Le travail de l'analogie proportionnelle consiste à mettre en rapport deux domaines différents (le 
corps humain et un système multi-média), puis à établir des correspondances terme à terme entre les deux champs sémantiques. Si le fil conducteur choisi est " pensée », par exemple, on pourra mettre en rapport cerveau et ordinateur. Si c'est « vue », on pourra rapprocher œil et caméscope, etc...

Par l'intermédiaire de la métaphore, nous passons donc d'un organe du corps (le cerveau) à la capacité de penser, du concret à l'abstrait. Cette capacité à représenter visuellement des abstractions est la raison première de l'utilisation des métaphores en publicité : elle permet de persuader le public tout en économisant de l'espace. L'analyse aristotélicienne selon le rapport d'analogie semble tout à fait suffisante pour expliquer comment cette persuasion opère. Le ratio aristotélicien montre bien en effet comment la métaphore crée une médiation entre une image et un concept, comment, grâce à ce que Paul Ricoeur nomme le «travail de la ressemblance » $(1976: 221-271)^{4}$ elle donne un visage à l'abstrait.

\section{La théorie de la substitution: fonction ostensive}

La rhétorique d'Aristote a servi de cadre de référence tout au long de l'antiquité grécoromaine et de la période scholastique, mais cette domination incontestée ne s'est maintenue qu'au prix de réévaluations successives, qui ont petit à petit réduit son empire à des questions de pure forme. C'est dans ce contexte que s'est développée la théorie substitutive de la métaphore, qui a vu son jour de gloire au dix-huitième siècle. Aristote, dans la Rhétorique, avait clairement marqué la prééminence de la métaphore sur la comparaison en définissant cette dernière comme une métaphore étendue :

Les comparaisons sont des métaphores, des métaphores qui demandent à être développées. (Rhétorique 1406b, 4, 20-25)

En revanche, à partir de Quintilien, on a réduit la métaphore à une comparaison abrégée, dans laquelle le motif de la comparaison a été gommé. Par exemple, dans la fameuse maxime de Hobbes: "L'homme est un loup pour l'homme", la cruauté, l'agressivité communes qui sous-tendent la métaphore ont été gommées. Il n'y a plus alors qu'un écart par rapport à l'usage courant, mais un écart réglé, qui peut toujours se réduire, ou s'expliquer exhaustivement ${ }^{5}$. Une métaphore n'apporte rien de plus que la paraphrase qu'on peut en faire. Son rôle est purement ornemental.

11 Dans cette optique, les publicitaires utilisent parfois des métaphores picturales ayant un sens conventionnel, simplement pour attirer l'attention du lecteur. Prenons par exemple la publicité pour les spectromètres de diffraction de la lumière à plusieurs angles publiée par la société Wyatt Technology dans le magazine LC GC Europe en octobre 2000 (annexe 2). L'image montre une alternance de cartes à jouer rouges et bleues formant une maisonnette, qui attire l'œil par le contraste des couleurs choisies, et intrigue le lecteur : que vient faire un château de cartes dans une revue dédiée à la chimie de séparation? L'effet de surprise semble venir de ce que seul l'élément figuré de la métaphore apparait sur l'image. Il faut lire l'argumentaire pour apprendre qu'en chimie on est souvent réduit à déterminer empiriquement les masses moléculaires des polymères, alors que les spectromètres de diffraction de la lumière à plusieurs angles permettent au contraire de mesurer ces masses de façon absolue. Le point commun entre un château de cartes et une mesure d'échantillon empirique se trouve donc être leur caractère précaire, leur manque de fiabilité, ce que souligne la conclusion du 
premier paragraphe : "You'll get measurements that are based on a solid foundation, not precariously dependent on someone else's work».

12 Les références à l'image sont claires, qu'elles apparaissent en positif ou en négatif (« solid foundations, precariously dependent »), mais ne supposent pas de complémentarité : le texte se suffit à lui-même et peut se comprendre indépendamment de son illustration.

13 Le rôle sémantique explicatif de la métaphore picturale dans la publicité semble ici réduit à sa portion congrue : énigme si on la considère en elle-même simple accroche photographique ou illustration redondante lorsqu'on la relie au texte qui l'accompagne. Dans cet usage ornemental des métaphores, la médiation est réduite à un minimum de caractéristiques communes (ici, la précarité, la non-fiabilité, par exemple), qui sont suggérées dans l'image, mais ne se révèlent vraiment que dans l'argumentaire publicitaire. Lorsque l'essentiel est simplement de donner à voir pour créer le désir de lire ou d'attirer l'attention du lecteur potentiel par une image énigmatique et séduisante, pour pouvoir ensuite le persuader par d'autres moyens dans le texte publicitaire, la théorie substitutive de la métaphore suffit tout à fait à en rendre compte.

\section{La théorie de l'interaction (Black) : fonction heuristique}

14 Par-delà la période de disgrâce du romantisme, la rhétorique connaît un renouveau certain depuis la deuxième moitié du vingtième siècle, entraînant dans son sillage un foisonnement d'approches nouvelles sur les métaphores. Dans l'optique de la médiation, la théorie la plus féconde me paraît être celle de l'interaction (Black 1962 \& 1979), parce qu'elle a le mérite de proposer une explication, d'éclairer le fonctionnement des métaphores en tant que produits ${ }^{6}$. Pour Max Black, une métaphore fonctionne comme interaction entre un foyer métaphorique figuré («focus») et un cadre littéral ( $f$ frame $)^{7}$. Certaines caractéristiques du foyer métaphorique se projettent sur le cadre littéral pour le réorganiser et, à l'inverse, le cadre littéral influe sur le foyer métaphorique. Les exemples analysés par Max Black sont tirés de la poésie ou de la vie quotidienne, mais sa théorie a également des applications dans les domaines scientifique et technologique ${ }^{8}$.

15 Pour expliquer la théorie de Max Black, prenons, par exemple, la publicité pour verres spéciaux de la firme Schott, publiée dans le magazine britannique New Scientist en septembre 1991 (annexe 3)9. L'image nous montre à la fois une feuille d'ortie exagérément grossie, dont on voit apparaître les poils irritants, et un morceau de conduit de cheminée en brique, dans lequel est fixé une gaine en verre "recusist ». Le contexte publicitaire ne laisse planer aucun doute sur l'identité des pôles de la métaphore. Dans la mesure où Schott est une firme spécialisée dans la fabrication de verres spéciaux ("number one in Europe for special glass », proclame fièrement sa devise), le verre «recusist» se trouve tout naturellement faire partie du cadre littéral. Cette appartenance sémantique est d'ailleurs reflétée par la présentation matérielle de l'image de la gaine, qui figure au-dessous de l'illustration, et en marge de l'argumentaire, comme pour faire corps avec lui. Par conséquent, la feuille d'ortie se trouve automatiquement jouer le rôle de foyer métaphorique. Dans ce contexte, où la 
distance entre les deux pôles de la métaphore semble grande, puisque nous passons du végétal au minéral et de la nature à la technique, l'interaction fonctionne sous forme d'un « voir comme » wittgensteinien (Wittgenstein 1961 : 325-361). Il s'agit de voir un conduit de cheminée comme un poil d'ortie, c'est-à-dire comme une vésicule contenant de l'acide formique, dont les parois durcies de calcium et de silice protègent le tissu végétal environnant :

In the stinging hairs there's a cell that contains a corrosive fluid. This is what stings us. It would harm the nettle too, but for deposits of calcium and silica which harden the cell wall and stop the fluid getting out.

Dans cette optique, la fonction d'une gaine de cheminée est vue sous un angle complémentaire: la perspective habituelle (permettre aux gaz de combustion de s'échapper à l'air libre au lieu d'enfumer la maison) est combinée à une préoccupation nouvelle (empêcher ces mêmes gaz de combustion d'attaquer ou d'encrasser le conduit de cheminée).

'Recusist' is impervious to gas and moisture. It stops corrosive condensation from the flue gases getting into the chimney wall and attacking the fabric of the building.

On voit clairement ici que la projection des caractéristiques habituellement attribuées aux poils d'orties (ou « lieux communs associés ", si l'on reprend le terme utilisé par Max Black), fait l'objet d'une sélection : le petit renflement au sommet du poil d'ortie, par exemple, n'entre pas en ligne de compte. Seuls apparaissent comme pertinents les traits décrivant le fonctionnement des vésicules urticantes : leur contenu corrosif, le durcissement de leurs parois, et l'imperméabilité qui en découle. L'observation du fonctionnement des poils d'orties, et le transfert de caractéristiques pertinentes dans le domaine des conduits de cheminée, permet de rendre compte du verre spécial « recusist ». À l'inverse, la marque de fabrication humaine se projette aussi, anthropomorphiquement, du verre "recusist» sur la feuille d'ortie, puisque nous voyons au premier plan, grossi au moins d'un facteur dix, une vésicule irritante, enserrée dans le tissu des cellules voisines, sous la forme d'une éprouvette tenue par une main verte.

18 Cet aller-retour entre le foyer et le cadre de la métaphore lui confère une fonction heuristique. La confrontation de deux domaines scientifiques aussi différents que la biologie végétale et la science des matériaux permet un éclairage nouveau, qui transforme notre perception des cheminées. Mais de quelle nature est cet éclairage ? S'agit-il d'une simple illustration pédagogique, pour mieux faire comprendre ce qui existe déjà, et peut être formulé en termes non-métaphoriques (ou moins métaphoriques), comme dans le cas du modèle planétaire de Rutherford ${ }^{10}$. qui présente l'atome comme un système solaire en miniature? S'agit-il plutôt d'un rapprochement intuitif qui génère une avancée technologique en mettant en relief de nouvelles similitudes?

En l'absence d'informations complémentaires, il est impossible de savoir si la métaphore du conduit de cheminée-poil d'ortie a été à l'origine de la création du verre «recusist » ou si elle illustre simplement, de façon adaptée et originale, les propriétés particulières d'un type de verre spécial. Dans ce cas ambigu, le "voir comme " peut se manifester soit dans un commencement, avec la perception intuitive soudaine d'un rapprochement créateur, d'une «médiation non-verbale » (Ricoeur, 1975 : 271), soit au 
contraire a posteriori, avec la construction d'une interaction calculée entre les deux pôles de la métaphore. Les deux stratégies tendent de toute façon au même but publicitaire : aider à comprendre pour persuader plus noblement. Et la théorie de l'interaction permet de voir comment cette stratégie de la persuasion par la découverte est mise en œuvre, en analysant comment certaines caractéristiques couramment associées aux orties sont projetées sur les verres spéciaux (pour conduits de cheminée). En créant ainsi des liens nouveaux entre deux domaines auparavant fort éloignés, la théorie de l'interaction modifie la compréhension que nous avons de ces deux domaines, étendant ainsi le travail de l'interaction jusqu'aux pôles de la métaphore.

\section{Conclusion}

Au terme de l'analyse des trois publicités pour Sony, Wyatt Technology et Schott, il apparaît que les trois théories de la métaphore présentées ici sont opérationnelles. L'hypothèse de départ selon laquelle toute théorie de la métaphore peut aider à comprendre la nature de la médiation à l'œuvre dans telle ou telle image publicitaire semble donc au moins partiellement confirmée, à condition de rendre explicite la stratégie de persuasion affichée. En effet, cette conclusion sur l'efficacité possible de théories, dont certaines sont largement jugées dépassées, ne peut prendre sens que dans un cadre pragmatique où les intentions des annonceurs et les effets produits sur les lecteurs sont pris en compte. La priorité peut alors être, selon les contextes, d'attirer l'attention du lecteur, de donner un visage concret à l'abstrait, ou encore d'aider à découvrir et comprendre. Dans ce cadre pragmatique, où les publicités sont considérées comme des produits de marché, la médiation métaphorique s'amplifie dans un "voir comme " qui englobe aussi bien le transport que le ratio aristotélicien, la perception intuitive que l'interaction. Le "voir comme" pointe vers cette perception du semblable dans le dissemblable qu'Aristote avait déjà notée, et qui permet de rendre compte de la métaphore en tant que processus créatif. Il ouvre donc l'espace d'une médiation, tout en proposant les moyens de structurer cet espace, c'est-à-dire d'expliquer et d'analyser cette médiation, en rendant compte cette fois de la métaphore comme produit fini.

\section{BIBLIOGRAPHIE}

Aldrich, Virgil. 1958. « Pictorial meaning, picture thinking, and Wittgenstein's theory of aspects ». Mind 67, 68-85.

Aristote. 1952. Poétique, J. Hardy trad. Paris : Les Belles Lettres.

Aristote. 1973. Rhétorique, livre III, Médéric Dufour et André Wartelle trad. Paris : Les Belles Lettres.

Black, Max. 1962. « Metaphor ». In Models and Metaphors. Ithaca, New York : Cornell University Press, 24-49. 
Black, Max. 1993. « More about metaphor ». In Ortony A. (dir), Metaphor and Thought. $2^{\mathrm{e}}$ édition corrigée. New York : Cambridge University Press, 19-41.

Boyd, Richard. 1993. " Metaphor and theory change. What is 'metaphor' a metaphor for? ». In Ortony A. (dir), Metaphor and Thought. $2^{\mathrm{e}}$ édition corrigée. New York : Cambridge University Press, 481-532.

Forceville, Charles. 1996. Pictorial Metaphors in Advertising. Londres : Routledge.

Gibbs, Raymond W. 1993. « Process and product in making sense of tropes » . In Ortony A. (dir), Metaphor and Thought. $2^{\mathrm{e}}$ édition corrigée. New York: Cambridge University Press, 252-276.

Gibbs, Raymond W. 1999. « Researching metaphor ». In Cameron L. and G. Low (dir.), Researching and Applying Metaphor. Cambridge : Cambridge University Press, 29-47.

Lakoff, George. 1986. « The meaning of literal ». Metaphor and Symbolical Activity 1, 291-96.

Lakoff, George \& Mark Johnson. 1980. Metaphors We Live By. Chicago, IL : University of Chicago Press.

Lakoff, George \& Mark Johnson. 1999. Philosophy in the Flesh, the Embodied Mind and its Challenge to Western Thought, Chicago, IL : University of Chicago Press.

Ricoeur, Paul. 1975. La métaphore vive. Paris : Seuil.

Schön, Donald. 1993. "Generative metaphors : a perspective on problem setting in social policy ». In Ortony A. (dir), Metaphor and Thought. $2^{\mathrm{e}}$ édition corrigée. New York : Cambridge University Press, 137-163.

Wheelright, Philip. 1962. Metaphor and Reality. Bloomington, IN : Indiana University Press.

Wittgenstein, Ludwig. 1961. Tractatus logoco-philosophicus, suivi d'Investigations philosophiques, P. Klossowski trad. Paris : Gallimard.

\section{NOTES}

1. Voir en particulier 1999 (46-47), pour une défense du caractère conceptuel des métaphores fondée sur les progrès des sciences cognitives («Johnson's theory of conflation... Grady's theory of primary metaphor... Narayanan's neural theory of metaphor... Fauconnier \& Turner's theory of conceptual blending ")

2. J'entends ici pragmatique dans le sens très large d'utilisation du langage dans son contexte.

3. La synecdoque est une figure de rhétorique où le tout (ici, le corps humain) est représenté par la partie (la tête).

4. L'étude de Paul Ricoeur dépasse très largement le cadre aristotélicien. Dans le cas de la publicité pour Sony, on pourrait bien sûr aussi interpréter le travail de la ressemblance à partir de la façon dont les sciences cognitives et l'informatique ont interprété la métaphore ordinateurcerveau, soit en projetant les caractéristiques de fonctionnement des ordinateurs sur le cerveau humain, soit, au contraire, en s'inspirant de notre connaissance des neurones et des synapses pour bâtir des systèmes d'intelligence artificielle.

5. Voir en particulier Ricoeur (1975: 28-40) pour un développement sur la genèse de la théorie de la substitution, et la différence entre métaphore et comparaison chez Aristote.

6. La reconnaissance d'une métaphore est un processus cognitif quasi-instantané, qui permet ensuite de les aborder comme "produits », c'est-à-dire d'étudier leurs effets, leur signification, leur interprétation. Pour la distinction entre processus et produit, voir Gibbs (1993 : 252-276, et 1999 : 37-44). 
7. La distinction littéral-figuré, dans le domaine des métaphores, présente certaines ambiguïtés. Lakoff, par exemple (1986: 292), ne détermine pas moins de quatre façons différentes de définir la littéralité. Forceville (1996: 29) admet certes le caractère fondamental de ces distinctions pour les métaphores mortes, mais estime que les tropes verbaux et picturaux des publicités s'assimilent plutôt à des métaphores vives, pour lesquelles la pertinence de l'opposition littéralfiguré reste pertinente. Je me situe ici dans cette perspective.

8. Voir en particulier Boyd (1993 : 481-532) et Schön (1975: 139-141), exemple développé cidessous.

9. Cette publicité date déjà d'une dizaine d'années, mais elle présente un argumentaire d'un type relativement rare, et dont je n'ai pas trouvé d'exemple plus récent.

10. Pour une discussion sur le caractère pédagogique de ce modèle, voir Boyd (1993 : 485-86). Il faut tout de même noter que ce modèle a eu à l'origine une fonction heuristique et a permis de rendre compte du fait que l'atome n'était pas plein, mais rempli de vide. En revanche, à partir du moment où les équations de Schrödinger ont remplacé la mécanique Newtonienne à l'échelle sub-atomique, le modèle de Rutherford a été dépassé, et a gardé un caractère purement pédagogique.

\section{RÉSUMÉS}

Cet article a pour but de tester l'utilité de diverses approches théoriques sur les métaphores, appliquées à l'analyse des images publicitaires. Pour la théorie comparative de la métaphore, qui remonte à Aristote, la médiation réside dans le «travail de la ressemblance » (Ricoeur 1976), et elle permet d'exprimer un concept abstrait par un objet concret (cf. le système memory stick de Sony). Pour la théorie de la substitution, qui a eu son jour de gloire à l'époque classique, une métaphore n'a de rôle qu'ornemental et la médiation consiste alors simplement à la «traduire » en termes littéraux. Dans cette optique, les publicitaires utilisent souvent les métaphores picturales comme accroches, pour attirer l'attention du lecteur (cf. le château de cartes de Wyatt Technology). La théorie interactive de la métaphore (Black 1962) amplifie au contraire la place de la médiation, en postulant que certaines caractéristiques du «foyer » métaphorique se projettent sur le «cadre » littéral qui l'entoure, et vice-versa. L'intérêt devient alors heuristique (cf. les verres spéciaux de Schott). Les résultats montrent donc que même des théories de la métaphore souvent considérées comme dépassées peuvent aider à analyser une image publicitaire, pourvu qu'elles soient en accord avec la stratégie de persuasion utilisée dans ce contexte particulier.

The purpose of this article is to test the usefulness of various theories of metaphor, when applied to advertisement picture analysis. Aristotle's comparison theory mediates via similitudes, allowing the characteristics of an abstract idea to be expressed through a concrete object (Sony's memory stick system). For the substitution theory of the classical period, metaphors are merely ornamental, and mediation is reduced to a "translation" into a literal paraphrase. Admen often use this ornamental feature to catch readers' attention (Wyatt Technology's house of cards). On the other hand, Black's interaction theory emphasizes mediation by defining metaphor as "mapping". Some of the characteristics of the metaphorical "focus" are projected onto the surrounding literal "frame", and vice-versa. This opens the door to discovery and heuristics (Schott's special glass). The results show that even old-fashioned theories of metaphor may help 
to analyse an advertisement picture, provided they are in keeping with the persuasion strategy used in that particular context.

INDEX

Keywords : advertisement, mediation, metaphor, picture, theory of metaphor Mots-clés : image, médiation, métaphore, publicité, théorie de la métaphore

\section{AUTEUR}

\section{MARIE-HÉLÈNE FRIES-VERDEIL}

Marie-Hélène Fries-Verdeil est maître de conférences à l'Université Grenoble 1, où elle enseigne l'anglais scientifique en DEUG de biologie et en école d'ingénieur. Ses centres d'intérêt en matière de recherche sont la rhétorique de l'image dans les publicités scientifiques et les métaphores. Elle est co-auteur de l'ouvrage Speaking Skills in Scientific English. Marie-

Helene.Fries@ujf-grenoble.fr 